\title{
Microcanonical Thermodynamics of First Order Phase Transitions studied in the Potts Model
}

\author{
D.H.E. Gross, A. Ecker and X.Z. Zhang * \\ Hahn-Meitner-Institut Berlin, Bereich Theoretische Physik, \\ 14109 Berlin, Germany \\ * Institute of Atomic Energy, Beijing, China
}

(November 14, 2017)

\begin{abstract}
Phase transitions of first and second order can easily be distinguished in small systems in the microcanonical ensemble. Configurations of phase coexistence, which are suppressed in the conventional canonical formulation, carry important information about the main characteristics of first order phase transitions like the transition temperature, the latent heat, and the interphase surface tension. The characteristic backbending of the microcanonical caloric equation of state $T(E)$ (not to be confused with the well known Van der Waals loops in ordinary thermodynamics or mean field approximations) leading to a negative specific heat is intimately linked to the interphase surface entropy.
\end{abstract}

Keywords: Microcanonical thermodynamics, phase transitions, surface tension.

Microcanonical thermodynamics describes the dependence of the volume $\Omega_{N}$ of the N-body phase-space of an interacting many-body system on the globally conserved quantities like the total energy, momentum, angular momentum, mass, charge etc.. This is the fundamental starting point of any statistical definition of thermodynamics. By Laplace transform of $\Omega_{N}(E)$ from the extensive quantities like the energy to intensive ones like inverse temperature $(\beta=1 / T)$ one obtains the more familiar Gibb's canonical partition function $Z(\beta)$. For systems interacting by short range twobody forces (with hard cores) both formulations are identical in the thermodynamic limit of infinitely many particles at the same particle density [1]. However, the two formulations are different for finite systems and more essentially are different even in their physical content for systems with long range forces like gravity [2] or Coulomb dominated systems see for example [3]. This will be discussed in a forthcoming paper.

One of the most interesting phenomena in thermodynamics are phase transitions. Computer simulations of simple models give a deep insight into the mechanism. Naturally these calculations can only be performed for small systems. The main characteristics like the transition temperature $T_{t r}$, the specific latent heat $q_{l a t}$, and the interphase surface tension $\sigma_{\text {surf }}$ are extrapolated to the infinite system. In the thermodynamic limit it should not matter whether the calculations for the finite systems are performed canonically or microcanonically. However, we will show for the two-dimensional, $q=10$ states, Potts model, which has a clear phase transition of first order from ordered to disordered spins, the extrapolations converge faster when started from the microcanonical-ensemble. Moreover, our investigation will clarify some of the most striking features of finite micro-ensembles, the occurence of the S-bend in the microcanonical caloric equation of state $T(E)$ and the occurence of a negative specific heat at a phase transition of first order, see e.g.: refs. 3 [ 8 . . \{Here we do not discuss the negative specific heat in thermodynamically unstable systems with long-range interaction as were discussed by Thirring [2]. The systems we discuss here are thermodynamically stable with short-range interactions. They behave properly in the canonical ensemble and have a positive specific heat there and in the thermodynamic limit.\}

The Hamiltonian of the Potts model is defined as

$$
H=\sum_{i<j}^{\prime}\left\{1-\delta_{\sigma_{i}, \sigma_{j}}\right\} .
$$

on a two dimensional lattice (here with periodic boundary conditions) of $N=L * L$ spins with $q=10$ possible values (components). The sum is over pairs of nearest neighbour lattice points only and $\sigma_{i}$ is the spin state at the i-th lattice point. For a microcanonical ensemble we have to calculate the partition sum over all possible different configurations $\nu$ with the same total energy $E$ :

$$
\Omega_{N}(E)=\sum_{\nu} \delta_{E_{\nu}, E}
$$

This is done by using the "Ergodic Microcanonical Metropolis Monte Carlo Algorithm" $(M M M C)$ [9, 10]. [The main idea here is to sample the energy in a narrow band $E-4 \leq E_{i} \leq E+4$ by Metropolis sampling. From the 
slope of $\left.\ln \left(P\left(E_{i}\right)\right)\right|_{E}$, in this energy-band one can determine $\left.\beta_{\text {micro }}(E)\right] . \Omega_{N}(E)$, the specific entropy $s(\epsilon)$, and the thermodynamic temperature are related by (we put Boltzmann's constant $k=1$ ) :

$$
\begin{array}{r}
N s(\epsilon)=\ln \left\{\Omega_{N}(E=N * \epsilon)\right\} \\
\beta_{\text {micro }}(\epsilon)=\frac{\partial s(\epsilon)}{\partial \epsilon} \\
T(\epsilon)=\frac{1}{\beta_{\text {micro }}(\epsilon)} .
\end{array}
$$

The connection to the canonical (Gibbs-) free energy $F(\beta)$ is via the Laplace transform in saddle-point approximation:

$$
\begin{array}{r}
Z(\beta)=\int_{0}^{\infty} \Omega_{N}(N \epsilon) e^{-\beta N \epsilon} N d \epsilon \\
\sim e^{N[s(\bar{\epsilon})-\beta \bar{\epsilon}]} T \sqrt{2 \pi N / c(\bar{\epsilon})} \\
\left.\frac{\partial}{\partial \epsilon} s(\epsilon)\right|_{\bar{\epsilon}}=\beta \\
F(\beta)=-T * \ln \{Z(\beta)\} \\
c(\bar{\epsilon})=-\left.\frac{\partial^{2} s}{\partial \epsilon^{2}} \frac{1}{\left(\frac{\partial s}{\partial \epsilon}\right)^{2}}\right|_{\varepsilon=\bar{\varepsilon}} .
\end{array}
$$

Plotting $s(\epsilon)$ and $\beta_{\text {micro }}(\epsilon)$ v.s. $\epsilon$ we see a convex intruder in $s(\epsilon)$ (fig.1), where $s(\epsilon)$ is reduced compared to its concave hull, the straight tangent line $\{\sim$ the canonical $s(\epsilon)\}$ by $\Delta s_{\text {surf }}$. For an infinite system van Hove's theorem 11,11 13 forbids the existence of any convex part in $s(\epsilon)$, because then the system gains entropy if it would divide spontaneously into two equal pieces one with ordered spins and with entropy $s_{1}=s\left(\epsilon_{1}\right)$, the other with disordered spins and entropy $s_{3}=s\left(\epsilon_{3}\right)$. Both pieces together would have the larger entropy $\bar{s}=\left(s_{1}+s_{3}\right) / 2 \geq s_{2}=s\left(\epsilon_{2}=\left(\epsilon_{1}+\epsilon_{3}\right) / 2\right)$. This is very nicely discussed by Hüller in several recent articles e.g. [14].

This argument does not apply to a finite system as the new surface of phase-separation reduces the specific entropy by $\Delta s_{p h-s e p}$ which is proportional to the number of lattice spins being fixed in the dividing surface i.e. $\Delta s_{p h-s e p} \propto$ $L / N \propto L^{-1}$. With growing size of the system, $L \rightarrow \infty, \beta_{\text {micro }}(\epsilon)$ approaches the horizontal line $\beta_{\text {can }}(\epsilon) \equiv \beta_{t r}$, determined by the Maxwell construction of equal areas $\mathcal{A}_{l}=\mathcal{A}_{r}$ on the left side below $\beta_{t r}$ and on the right above it, lower half of Fig.1.. On the other hand the surface entropy $\Delta s_{\text {surf }}$ is equal to one of these areas $\mathcal{A}$, half of the shaded area under the S-like oscillation of $\beta_{\text {micro }}(\epsilon)$ between the curve and the line $\beta_{t r} . \beta_{\text {micro }}(\epsilon)$ can directly be calculated by $M M M C$ so also the area $\mathcal{A}=\Delta s_{\text {surf }}$. As the Potts model has only nearest neighbour interactions the interphase surface tension $\sigma_{\text {surf }}=T * \Delta s_{\text {surf }}$ consists of an entropy part only. As the entropy of the special configuration with planar phase-separation can only be $\leq s(\epsilon)$ we have $\Delta s_{\text {surf }} \leq \Delta s_{\text {ph-sep }}=O(1 / L)$. We make the conjecture that the area $N * \mathcal{A}$ under $N * s(\epsilon)$ scales like the cross-section of the lattice $(\propto L)$.

The intimate link of $\mathcal{A}=\Delta s_{\text {surf }}$ to the surface tension can also be seen from the way how the surface tension is calculated in conventional canonical Monte Carlo simulations e.g. 115 17]: Here the probability $P_{T}(\epsilon)$ to find the system with temperature $T$ at an energy $\epsilon$ shows at the transition temperature two well separated maxima with a deep valley in between. The ratio of the minimum to the maximum is by [15] equal to:

$$
N * \Delta s_{\text {surf }}=-\ln \left\{\frac{P_{t r}(\min )}{\sqrt{P_{t r}(\max 1) P_{t r}(\max 2)}}\right\}
$$

Fig. 2 shows $L * \Delta s_{\text {surf }}$ vs. the inverse lattice size $1 / L$ in comparison with the surface tension determined by the multi-canonical method [18]. (Actually the quantity shown is twice the surface tension as we use periodic boundary conditions and consequently two cuts have to be made to separate the two phases.) Both results scale to the analytically known asymptotic limit 16,18. We make an interesting observation here : The microcanonical surface tension depends much less on the size of the lattice than the canonical one. That means one may get its asymptotic value already at smaller sizes of the system than canonically.

Insight into the possible mechanism leading to this weaker finite size scaling is obtained from the projected correlation function $g^{(1)}(d x)[19]$.

$$
g^{(1)}\left(d x=\left|i_{x}-j_{x}\right|\right)=\frac{1}{L} \sum_{i_{y}, j_{y}}\left\{\delta_{s_{i}, s_{j}}-\frac{1}{q}\right\} \cos \left\{\frac{2 \pi}{L}\left(i_{y}-j_{y}\right)\right\}
$$


In the canonical ensemble it approaches

$$
\begin{array}{r}
g^{(1)}(d x) \propto \cosh \left\{(d x-L / 2) / \xi^{(1)}\right\} \\
\xi^{(1)}=\xi / \sqrt{1+(2 \pi \xi / L)^{2}}
\end{array}
$$

with $\xi$ the correlation length of the infinite lattice [19]. Figure 3 shows $g^{(1)}(d x)$ at an excitation energy $\epsilon=0.7$ in the middle of the coexistence region. In the microcanonical ensemble $g^{(1)}$ does not need to be an exponential-like function and in fact it drops down faster in the region of coexistence $(0.33 \leq \epsilon \leq 1.05)$. In any case, the region of coexistence of both phases, gets suppressed for large $N$ by the Laplace transform eq.(6). It does not contribute because in this region there is no stationary point, no solution of the equation (8) and all information contained in events showing the coexistence of both phases is lost in the canonical ensemble. This will more thoroughly be investigated in a forthcoming publication.

Most of these calculations were done on different workstations. For the largest lattice $(L=100)$ we performed $3.2 * 10^{6}$ sweeps. To destroy correlations due to the previous energy step the spins in an arbitrarily chosen rectangular part of the lattice were first uniformly lifted one value higher up (cyclic) and the energy was restored by switching random spins of the lattice. This procedure has the advantage that the perturbation per latice point is $\propto 1 / L$ and all correlations inside the rectangle are conserved. In between these macroscopic moves the spins were individually updated in sequence one after the other. As can be seen from fig. 1 the accuracy for $L=100$ is not optimal but sufficient for the demonstration of the method. Due to bad computational conditions here at the HMI it was not yet possible to do better.

An important lesson can be learned here : Following M. Fisher [20] a phase transition of first order is distinguished from a transition of second order by a non-vanishing interphase surface tension. That means the microcanonical caloric equation of state for a phase transition of first order must show an S-oscillation and consequently a negative specific heat $c=\partial \epsilon / \partial T$. This solves the outstanding problem of the backbending and of the negative specific heat at a phase transition of first order in the microcanonical ensembles discussed before by many authors e.g. ref. [2,6 8]. It also appears at the condensation phase transition of selfgraviting astrophysical bodies which again must be treated microcanonical as the long range of gravity does not allow for the transition to the thermodynamic limit [2]. \{A selfgraviting system at constant energy behaves microcanonically quite differently from the same system at constant temperature (canonical). Whereas in the second case it can totally implode at low temperatures and convert all surplus energy to the heatbath, in the first case it can only partly implode because all energy must be stored in the noncollapsed rest of the system.\}

Figure 4 shows the three crossing points $\epsilon_{1}, \epsilon_{2}, \epsilon_{3}$ of the Maxwell line $\beta_{t r}$ with $\beta_{\text {micro }}(\epsilon)$ as function of the inverse lattice length $1 / L$. The difference $q_{l a t}=\epsilon_{3}-\epsilon_{1}$ at $L=\infty$ is the specific latent heat of the transition, which is analytically known from Baxter 21]. Our results up to the lattice length $L=100$ agree well when extrapolated linearly in $1 / L$ to $L=\infty$. Only the middle solution of $\beta_{\text {micro }}\left(\epsilon_{2}\right)=\beta_{t r}$ fails. However, this point is numerically as well as also physically the instability point. For the latent heat it is irrelevant. Again the finite size scaling of the microcanonical $\epsilon_{i}$ turns out to be very weak. It is rather surprising to find this simple scaling at such small lattices. As the theory of finite-size scaling for the microcanonical ensemble does not yet exist we have to take this promising result with care. However, as was discussed by Hüller [22] this may be at no surprise, as much of the size dependence of the canonical ensemble comes from the trivial factor $N$ in the exponent of the Laplace transform (6) which has nothing to do with the physics contained in the specific entropy $s(\epsilon)$. In a future publication we will discuss possible reasons for this weak sensitivity of the microcanonical Potts model to the finite size of the lattice and we will compare the microcanonical correlation functions with the canonical ones.

We believe our findings with the $q=10$-states Potts model are characteristic for all microcanonical ensembles. In fact the backbending of the caloric equation of state $T(E)$ was found in many other finite system e.g. fragmenting nuclei [3], fragmenting atomic clusters 㕶, "melting" phase transitions in van der Waals clusters [8.5] . More work, however, must be done to understand what the "surface" entropy, the area $\mathcal{A}$ under the S-shape of $\beta_{\text {micro }}(\epsilon)$, means in these cases which cannot be extrapolated to infinite systems. The scaling property of the microcanonical ensemble is yet unknown and must be examined.

We want to thank the Fachbereich Physik of the Freie Universität Berlin and the Institut für Hochenergiephysik, DESY, Zeuthen for the excellent computing conditions. Only with their generous support we were able to perform these numerical calculations. 
[1] L. van Hove. Quelque propriétés générale de l'intégrale de configuration d'un système de particules avec interaction. Physica 15 (1949) 951.

[2] W. Thirring. Systems with negative specific heat. Z. f. Phys. 235 (1970) 339-352.

[3] D. H. E. Gross. Statistical decay of very hot nuclei, the production of large clusters. Rep.Progr.Phys. 53 (1990) 605-658.

[4] D. H. E. Gross and P.A. Hervieux. Statistical fragmentation of hot atomic metal cluster. Z. Phys. D 35 (1995) $27-42$.

[5] R.M. Lynden-Bell. Landau free energy, landau entropy, phase transitions and limits of matastability in an analytical model with a variable number of degrees of freedom. to be pulished in Molecular Physics, 1995.

[6] R.M. Lynden-Bell. Negative specific heat in clusters of atoms. to be published in Galactic Dynamics, 1995.

[7] J.P.K. Doye and D.J. Wales. An order parameter aproach to coexistence in atomic clusters. J. Chem. Phys.102 (1995) 9673-9688.

[8] D.J. Wales. preprint, Cambridge, 1995.

[9] Koo-Chul Lee. Rejection-free monte carlo technique. J. Phys. A: Math. Gen.28 (1995) 4835-4842.

[10] Xiao ze Zhang and D. H. E. Gross. An ergodic monte carlo method for simulating the microcanonical ising model with finite size. In The Second International Conference on Computational Physics ICCP-2, volume 2, page 530, Beijing, China, 1993.

[11] S. Katsura and H. Fujita. Progr.Theor.Phys. 5 (1950) 997.

[12] S. Katsura. J.Chem.Phys. 22 (1954) 1277.

[13] T.L. Hill. J. Phys. Chem. 23 (1955) 812.

[14] A. Hüller. First order phase transition in the canonical and the microcanonical ensemble. Z.Phys.B 93 (1994) $401-405$.

[15] K. Binder. Monte carlo calculation of the surface tension for two- and three-dimensional lattice-gas models. Phys. Rev. A 25 (1982) 1699-1709.

[16] C. Borgs and W. Janke. An explicit formula for the interface tension of the 2d potts model. J. Phys. I, France 2 (1992) 2011-2018.

[17] W. Janke. Interface tension and correlation length of $2 \mathrm{~d}$ potts models: Numerical versus exact results. Int. Journ. Mod. Phys. C 5 (1994) 75.

[18] A. Billoire, Th. Neuhaus, and B.A. Berg. A determination of interface free energies. preprint Saclay, SPhT-93/065, 1993.

[19] W. Janke and S. Kappler. Correlation function at $\beta_{t}$ in the disordered phase of 2d potts models. Phys. Lett. A 197 (1995) 227-234.

[20] M. E. Fisher. Physics 3 (1967) 255.

[21] R.J. Baxter. J. Phys. C6 (1973) L445.

[22] A. Hüller. Finite size scaling at first order phase transitions ? Z.Phys.B 95 (1994) 63-66. 


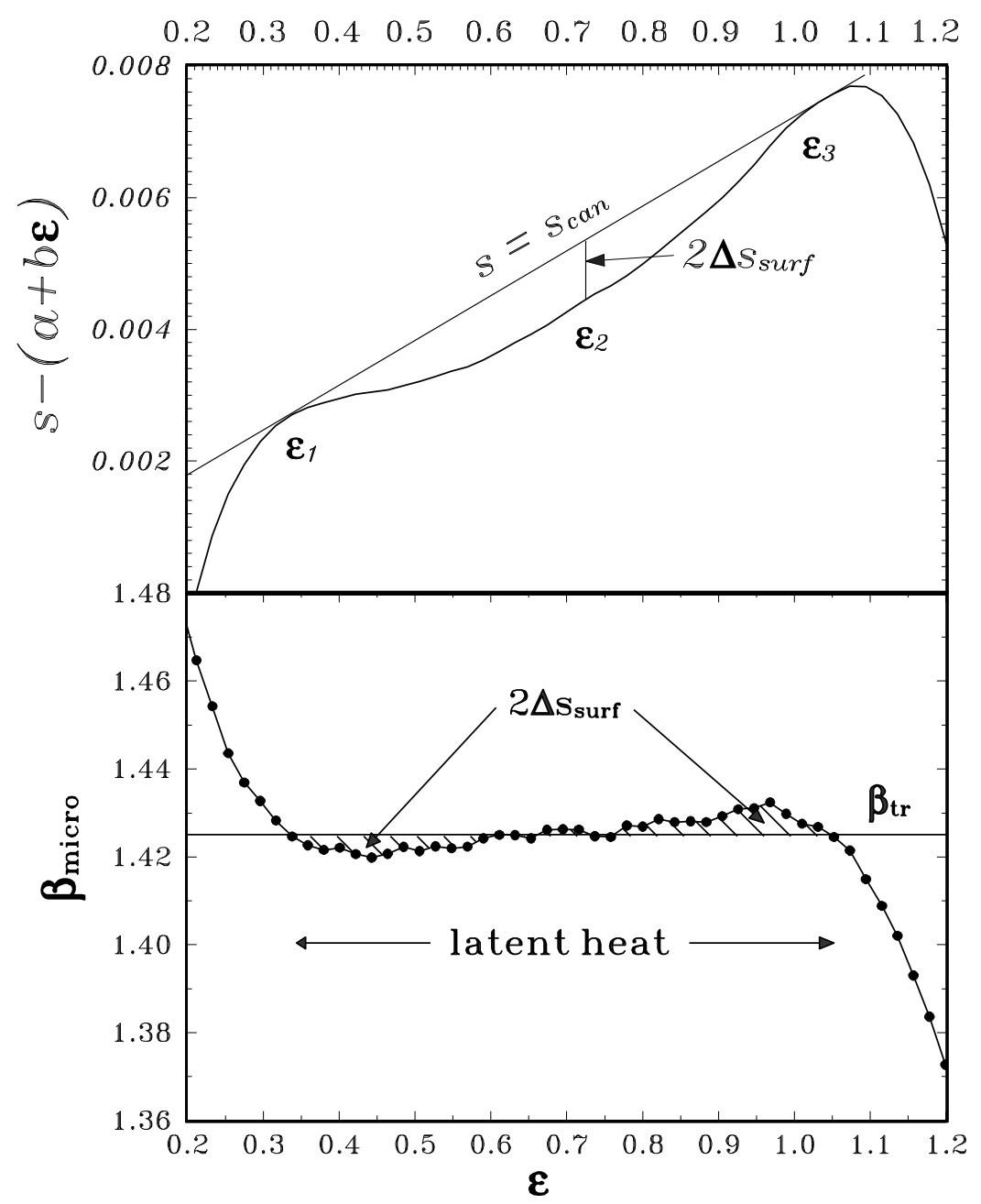

FIG. 1. a) Specific entropy $s(\epsilon)=\int_{0}^{\epsilon} \beta_{\text {micro }}(\bar{\epsilon}) d \bar{\epsilon}$ vs. the specific energy $\epsilon$ for the 2-dim. Potts model with $q=10$ on a $100 * 100$ lattice. In order to visualize the anomaly of the entropy the linear function $a+b \epsilon(a=s(0.2119), b=1.4185)$ was subtracted. Because we use periodic boundary conditions one needs two cuts to separate the phases and the convex intruder is twice the surface-entropy.

b) Inverse temperature $\beta_{\text {micro }}(\epsilon)=1 / T(\epsilon)$ as directly calculated by $M M M C$ 


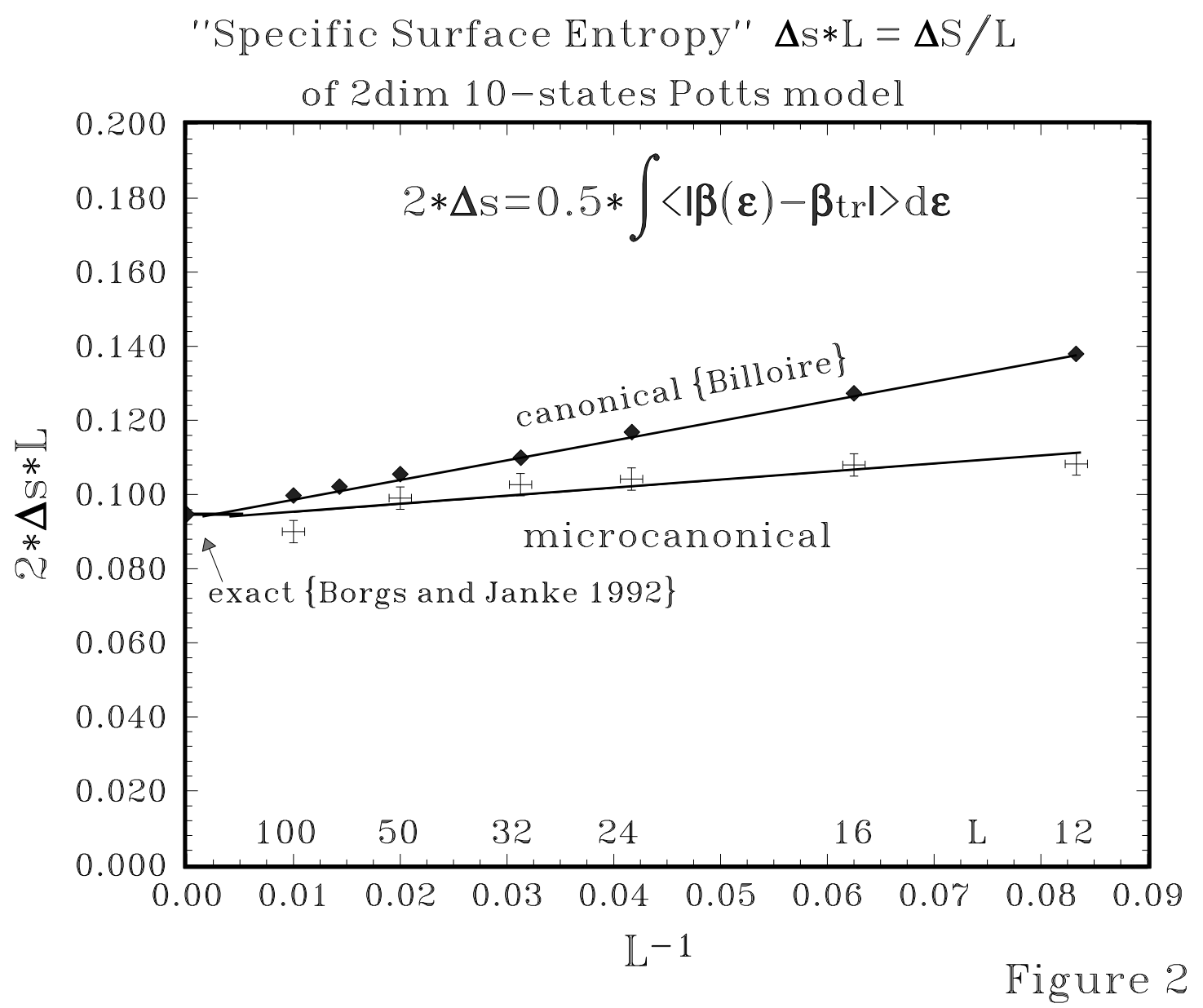

FIG. 2. Surface entropy $2 \Delta s_{\text {surf }}$ evaluated as half the shaded area in figure 1 as function of the inverse lattice length $1 / L$ compared to the same quantity from the multi-canonical method of Billoire 18]. 


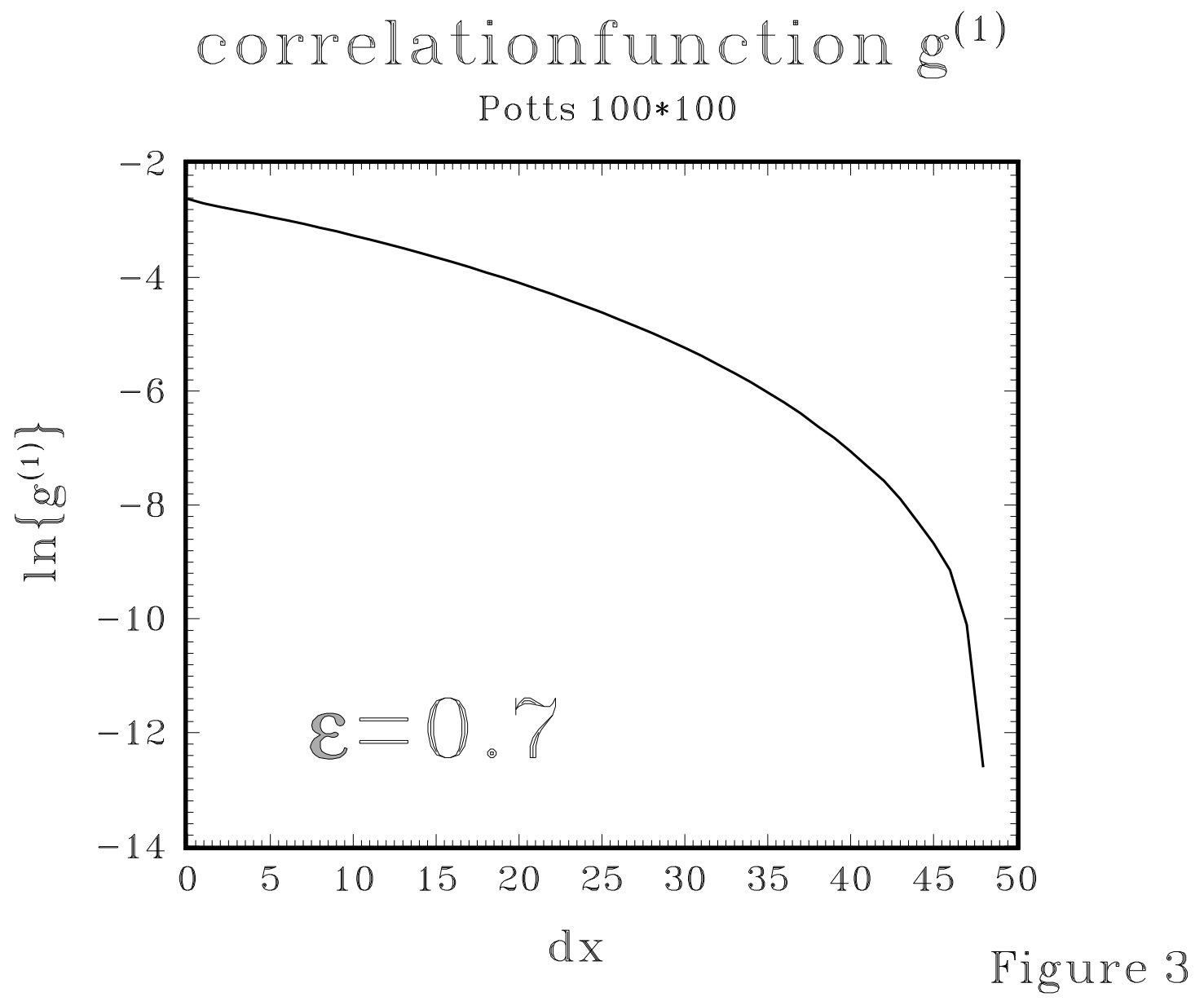

FIG. 3. The microcanonical correlation function $g^{(1)}(d x)$ at $\epsilon=0.7$ in the coexistence region. 


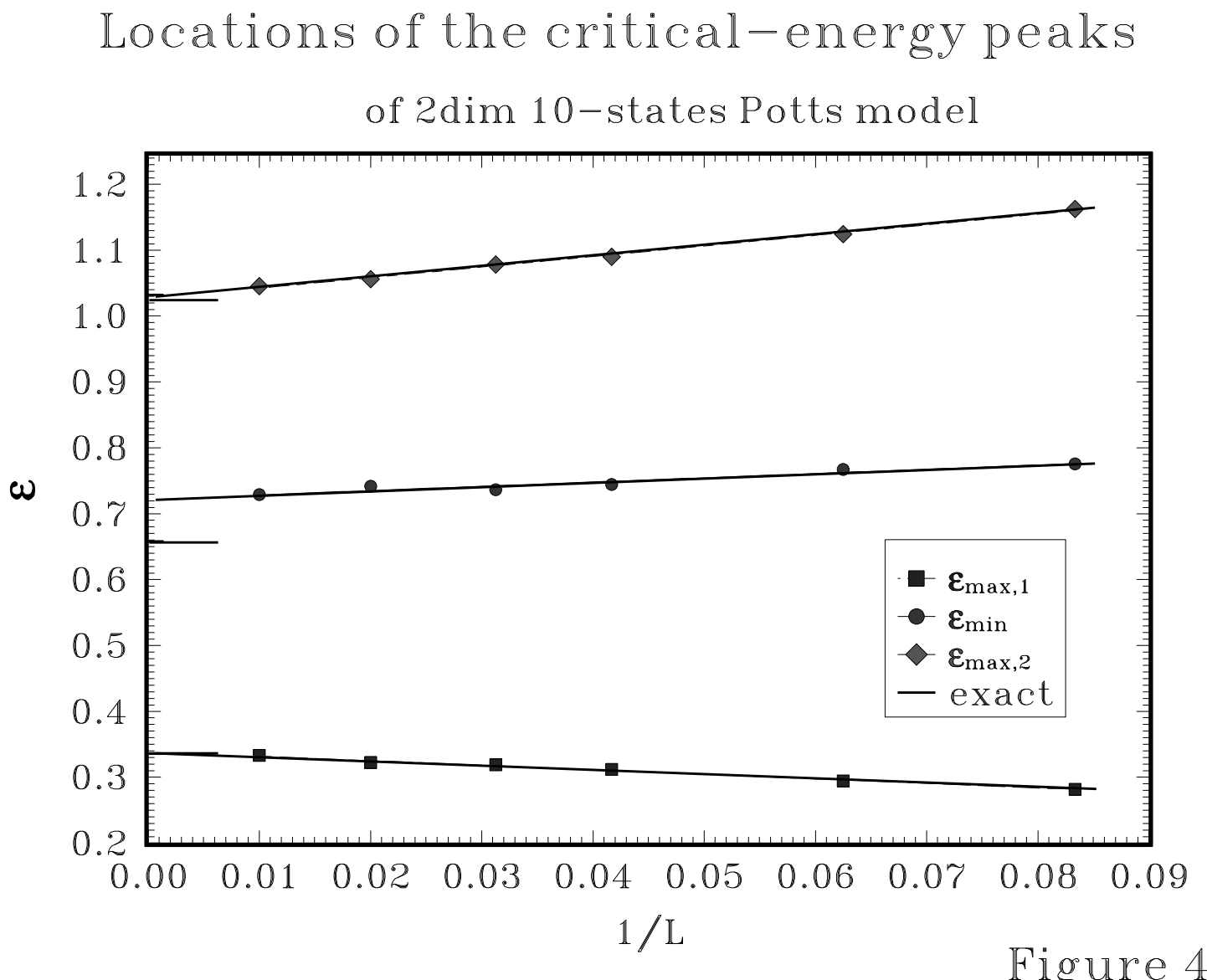

FIG. 4. The three solutions of $\beta_{\text {micro }}\left(\epsilon_{1,2,3}\right)=\beta_{t r}$ as function of the inverse lattice length $1 / L$. 